\title{
A design of HDPE flexible spline of harmonics gear
}

\author{
Pholchai Chotiprayanakul, Nattakul Khamsri and Neeracha Kumjaroen \\ Industrial Engineering Department, Faculty of Engineering, \\ King Mongkut's Institute of Technology Ladkrabang, Bangkok, Thailand
}

\begin{abstract}
In a design of a small robot, selecting a servo-motor is the most important method in designing process. Common miniature servo motors for small robots normally use conventional gear train set or planetary gear set. Gear train and planetary gear give very low gear ratio whereas its weight is too heavy. On the other hand, Harmonic gear system, which is developed from strain wave drive gearing, gives better the highest gear ratio per weight than those two gear systems. In this paper, a plastic flexible spline gear is presented in order to replace a thin metal spline. The plastic flexible spline gear is designed under gear physical requirement and servo-motor properties. The spline thicknesses are varied in a range of 1 to 4 millimetre and simulations on strength, fatigue, and torque requirement are made to verify the designs.
\end{abstract}

\section{Introduction}

There are many brands of servo-motors for a small robot, all of them contain with gear train sets. A robot joint normally needs a servomotor provide speed about $30 \mathrm{RPM}$ and torque depending on the size of the robot. For DC motor running at 1,000 to 3,000 RPM, a gear set must have gear ratio of $1: 33$ to $1: 100$. In 1957, C.W. Musser introduced a strain wave gear system, which is now known as harmonic gear system. The harmonic gear set provides high gear ratio and low weight and it is generally used in industrial robot today. Analysis and Design of Harmonics Gear presented by Lih-Min Hsia [1] shows a guide line to consider the geometry of the harmonic gear. Sensor monitoring of torque and force in the harmonics gear is presented in [2], forces detect and formulate a force function. Many novel types of the harmonics [3-4] were introduced for industrial use. In a household robot, servo-motor may not be need as strong as the servo-motor in the industrial robot thus a plastic flexible spline for harmonic gear set in servo-motor is considered to replace a metal spline. The harmonic gear designing method can be separated into 4 steps: first gear geometry design and then second strength consideration. After spline expansion force is

\footnotetext{
*Pholchai Chotiprayanakul: kcpholch@kmitl.ac.th
}

calculated, torque to roll the flexible spline is formulated in this third step. At final step, the flexible spline fatigue is simulated.

\section{Harmonic Gear Geometry}

A harmonic gear set consists of 3 parts; 1) Circular Spline, 2) Flexible Spline, and 3) Wave Generator and the geometry of harmonic gear includes number of gear teeth, the gear tooth shape, diameter of circular spline, and thickness of the flexible spline.

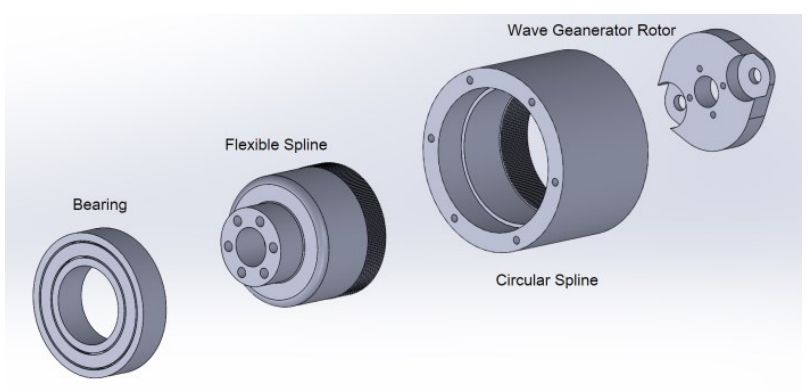

Figure 1 Components of Harmonics Gear

Harmonics gear reduces rotation speed by the uneven number of the teeth between the flexible spline and the circular spline. By number of teeth, diameter of the flexible spline is slightly smaller than the circular spline. The wave generation rotor will stretch the flexible 
spline shell along a diameter line until teeth of both splines attach. When the wave generation rotor rolls, contact points of the attached teeth will move around. After the wave generation rotor rotates a cycle, the flexible spline will shift couple teeth depending on different number of teeth between flexible and circular spline. With 1:100 gear ratio configuration, the number of gear teeth is considered from strain wave gear ratio equation as show in (1) where CST is number of circular spline teeth and FST is number of flexible spline teeth.

$$
\text { Gear ratio }=\frac{C S T-F S T}{C S T}
$$

The smallest number of teeth will be 99 teeth of flexible spline and 100 teeth of circular spline but this configuration has only a contact point. Single contact point, the wave generator touches only a point on the flexible spline makes the wave generator is unbalanced and shakes the whole body of gear. Avoiding balancing issue, 2 contact points flexible spline is considered, the gear will have 198 teeth of flexible spline and 200 teeth of circular spline.

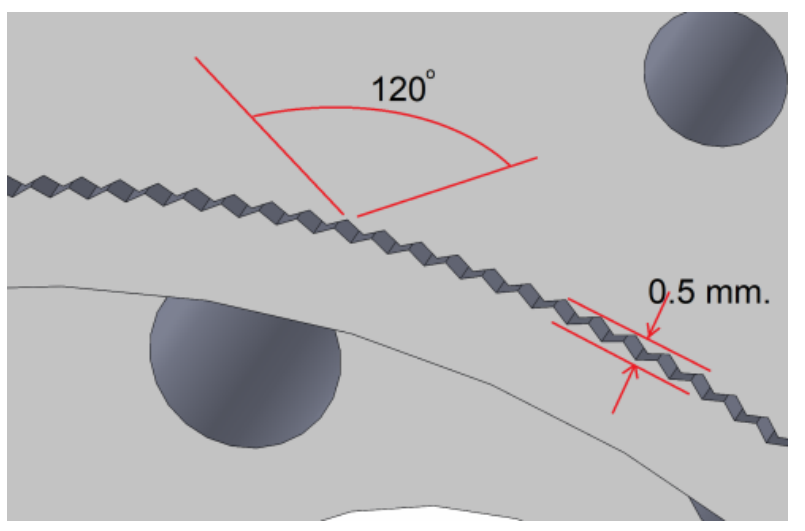

Figure 2 Harmonics Gear's Teeth Geometry

Simply triangle tooth shape is chosen with 32 teeth per inch thread. Tooth's vertex is at 120 degree and its height is $0.5 \mathrm{~mm}$. For 10 millimetres of a tooth length, the shear strength is about $23 \mathrm{MPa}$ and ultimate shear force per tooth will be $398 \mathrm{~N}$. Thus the payload that the harmonic gear set is able to support will be 19.8 N.m.

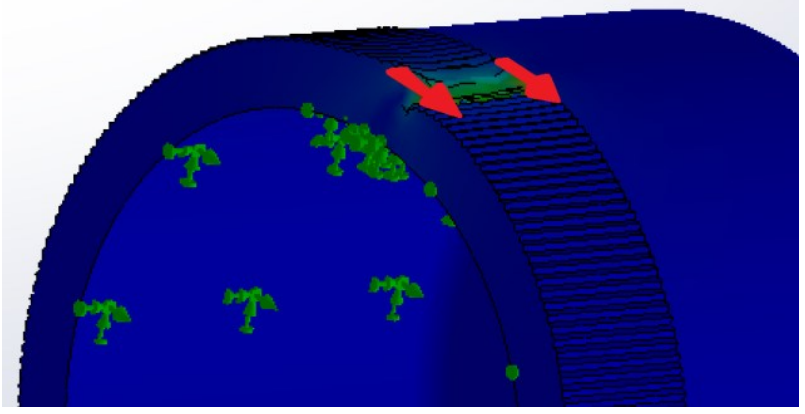

Figure 3 Simulation of Teeth Strength and Torque Load

This tooth shape configuration at 198/200 teeth spline will give the inside diameter of circular spline at $50.53 \mathrm{~mm}$. and the outside diameter of flexible spline at $50.03 \mathrm{~mm}$. thus the stretching distance of flexible spline is $0.5 \mathrm{~mm}$. The thickness of flexible spline will define the stretching force, which lead to the loss of motor's torque. For prelim study, researchers define for 4 thicknesses as 1.3 to $4.3 \mathrm{~mm}$. of the flexible spline shell to find out the optimal thickness which gives minimum torque loss and maximum life cycle.

\section{Stretching Force And Life Cycle Estimation}

The force to stretch the flexible spline is considered in this section. The size of force depends on thickness of the flexible spline varied from 1.3 to $4.3 \mathrm{~mm}$. and what material the flexible spline made of. High-Density PolyethylEne (HDPE) is selected to make the flexible spline. HDPE tensile strength is around $32 \mathrm{MPa}$ and flexural modulus at $1.25 \mathrm{GPa}$. Stretching force is simulated on a CADsoftware which the flexible spline expansion distance at $0.5 \mathrm{~mm}$. is a control parameter. The result of simulation shows the stretching force is increasing when the flexible spline shell is more thicken. 


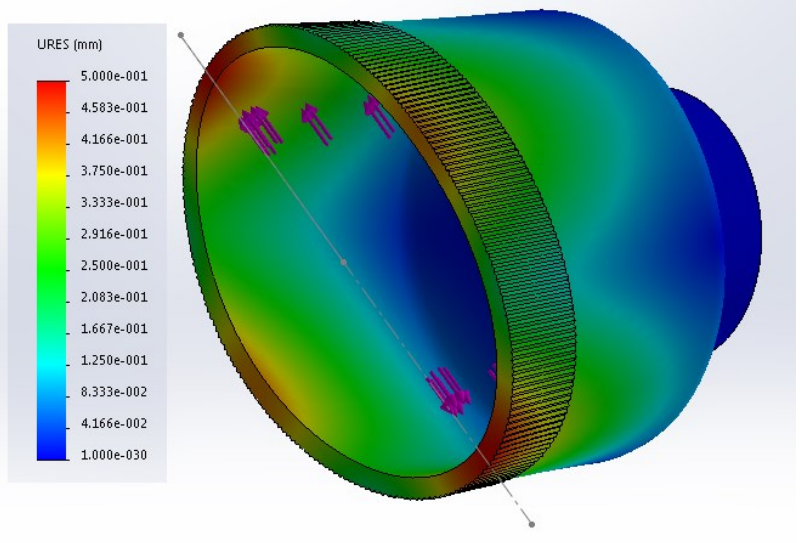

Figure 4 Simulation of Stretching Force at $0.5 \mathrm{~mm}$. Displacement

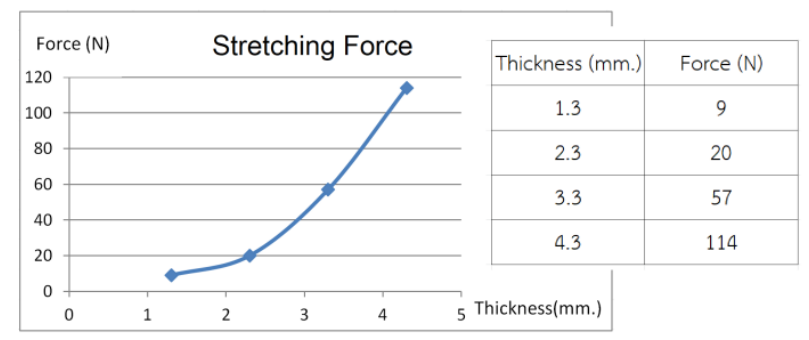

Figure 5 Stretching Force and Flexible Spline Thickness

The harmonic wave generator rolls inside the flexible spline and creates a repeating stretching force on the flexible spline. This cycle load may conduct fatigue on the flexible spline. From [5], researchers provide a S-N formula to estimate the life cycle for HDPE as shown in equation 2 .

$$
S=32.24 \times N^{-0.068}
$$

Where $S$ is stress on workpiece and $N$ is the life cycle. We ran a simulation with thickness series from $1 \mathrm{~mm}$. to $5.5 \mathrm{~mm}$. step $0.5 \mathrm{~mm}$. of the flexible spline. The maximum stresses of the stretching flexible splines are collected from simulation tests and shows in Figure 6.

The result shows relation between life cycle and thickness of flexible spline. The stress of stretching flexible spline depends on the thickness. However, the stress at $3-3.5 \mathrm{~mm}$. of the flexible spline thickness is on critical condition that is the stress closed to the yield strength of the HDPE material. The cup shape of the flexible spline has started to deform in the plastic mode and may not full recover spring back. Thus, we do only focus on 1.3 $\mathrm{mm}$. thickness that provides a low stretching force and thinnest flexible spline's shell which can be built. The life cycle of this thickness will be over 296 billion cycles.

\begin{tabular}{|c|c|c|}
\hline Thickness $(\mathrm{mm})$. & Stress $(\mathrm{MPa})$ & Life Cycle \\
\hline 1 & 5.28 & $3.58 \mathrm{E}+11$ \\
\hline 1.5 & 5.35 & $2.96 \mathrm{E}+11$ \\
\hline 2 & 5.52 & $1.87 \mathrm{E}+11$ \\
\hline 2.5 & 7.05 & $5.13 \mathrm{E}+09$ \\
\hline 3 & 8.52 & $3.15 \mathrm{E}+08$ \\
\hline 3.5 & 17.33 & $9.22 \mathrm{E}+03$ \\
\hline 4 & 19.29 & $1.91 \mathrm{E}+03$ \\
\hline 4.5 & 21.72 & $3.33 \mathrm{E}+02$ \\
\hline 5 & 24.25 & $6.59 \mathrm{E}+01$ \\
\hline 5.5 & 26.46 & $1.83 \mathrm{E}+01$ \\
\hline
\end{tabular}

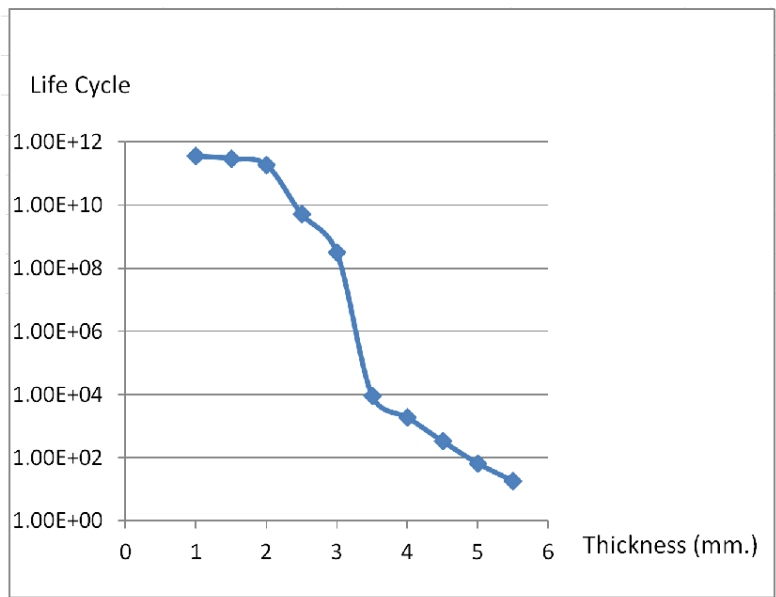

Figure 6 Thickness of Flexible Spline and Stress vs. Life Cycle

\section{Stall Torque}

When harmonic gear is used in a servo-motor set, the stretching flexible spline will resist the motor to roll. The resistance is known as stall torque load. Stall torque related on the stretching force and the radius of the flexible spline.

$$
T_{s}=2 \pi R F_{S}
$$

where $T_{s}$ is stall torque, $R$ is radius of the flexible spline and $F_{s}$ is the stretching force. Stall torque would be 2.8 N.m. thus the servo- 
motor must has torque greater than stall torque (2.8 N.m.) but it should not excess over the payload torque (19.8 N.m. from topic 2) multiply by gear ratio $(1 / 100)$. The servo-motor torque will be around 3 N.m.

\section{Prototyping}

After all configurations of the flexible spline is studied and selected as shown topics above. All 3 components, which include circular spline, flexible spline, and wave generator rotor, are made for a prototype of harmonics gear system. Circular spline is made with steel for the first prototype but we plan to replace it with a plastic circular spline in the future work. The wave generator rotor is made by $3 \mathrm{D}$ printing process and assembly with 2 roller bearings and an aluminium shaft. The flexible spline is made by the injection molding process thus a mold that has a cavity of flexible spline shape was design and built before injecting melt HDPE into the mold.

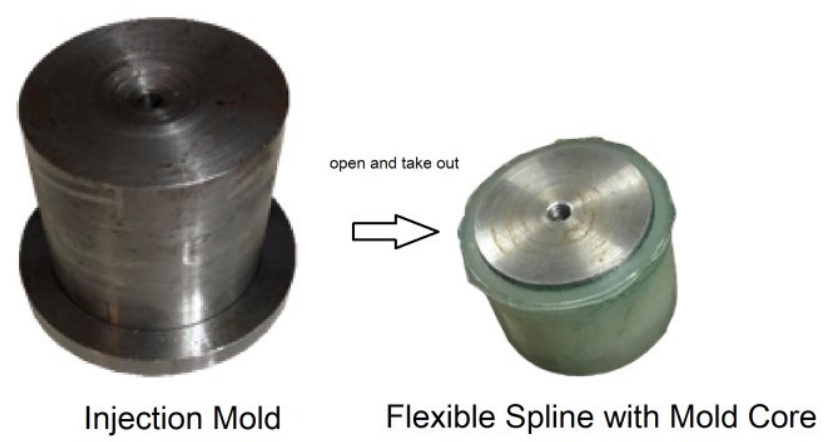

Figure 7 Injection Mold for Flexible Spline

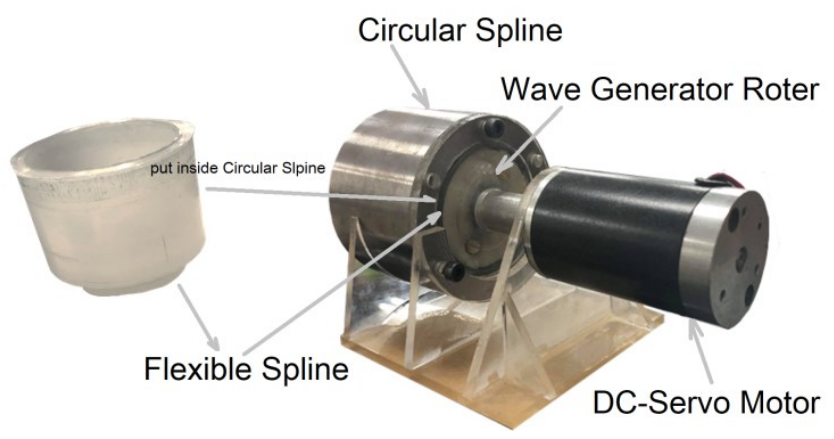

Figure 8 The 1:100 Harmonics Gear System Prototype

\section{Conclusion}

This paper shows a design of HDPE plastic flexible spline of harmonic gear. Strength of gear tooth, forces, fatigue and life cycle are considered with simulation tests. A prototype of the harmonics gear system is built to verify our design. This research needs to extend more experiment to find actual gear ratio, actual stall torque, and the life of the flexible spline.

\section{Reference}

1. Lih-Min Hsia, The Analysis and Design Of Harmonic Gear Drives (1988), Proceedings of the 1988 IEEE International Conference on Systems, Man, and Cybernetics, 8-12 Aug. 1988.

2. I. Godler, M. Hashimoto, Torque control of harmonic drive gears with built-in sensing (1998), Proceedings of the 24th Annual Conference of the IEEE Industrial Electronics Society, vol: 3, pp.1818-1823, Aachen, Germany 31 Aug-4 Sep 1998

3. Jan Rens, Kais Atallah, Stuart D. Calverley, and David Howe, A Novel Magnetic Harmonic Gear (2010), IEEE Transactions On Industry Applications, Vol. 46, No. 1, January/February 2010

4. Jonathon W. Sensinger and James H. Lipsey, Cycloid vs. Harmonic Drives for use in High Ratio, Single Stage Robotic Transmissions (2012), IEEE International Conference on Robotics and Automation, River Centre, Saint Paul, Minnesota, USA May 14-18, 2012

5. A. Aid1; M. Bendouba, A. Talha, N. Benseddiq, M. Benguediab, and S. Zengah, Uniaxial Fatigue of HDPE-100 Pipe. Experimental Analysis (2014), Directory of Open Access Journals in Engineering, Volume 4, Number 2, pp. 600-604(5), 2014 